\title{
MULTICOHERENCE TECHNIQUES APPLIED TO INVERSE LIMITS $\left({ }^{\mathbf{1}}\right)$
}

\author{
BY \\ SAM B. NADLER, JR. \\ Dedicated to my wife, Elsa
}

\begin{abstract}
Sufficient conditions are given so that the multicoherence degree of continua is not raised when taking inverse limits. These results are then applied to inverse limits of special types of spaces.
\end{abstract}

1. Introduction. In $\S 3$ we prove a structure theorem (Theorem 6) for arcwise connected inverse limits of circles (with onto bonding maps). We use results announced in [11] that relate the theory of multicoherence to inverse limits. In $\$ 2$ we sketch proofs of some of the results in [11].

Throughout this paper a continuum is a compact connected Hausdorff space containing more than one point. For the notation and terminology relating to inverse limits, see [4] and [7]. In particular, $\Pi_{\lambda}$ denotes projection from the inverse limit space into the $\lambda$ th factor space. All notation and terminology in this paper pertaining to multicoherence or to property (b) can be found, for the metric case, in [14]. Since the results we use from [14] are valid for (nonmetric) continua without essential changes in the proofs, we shall frequently refer to results in [14] as though they were stated for the more general class of continua.

2. Multicoherence and inverse limits. If $X$ is a continuum, then we say that $X$ has multicoherence degree $k[14$, p. 83$]$ provided

lub $\left\{r\left(X_{1}, X_{2}\right): X_{1}\right.$ and $X_{2}$ are subcontinua of $X$ with $\left.X_{1} \cup X_{2}=X\right\}=k$, where $r\left(X_{1}, X_{2}\right)$ denotes the number of components of $X_{1} \cap X_{2}$ less one. The multicoherence degree of $X$ is denoted by $r(X)$. We note that $r(X)=0$ is equivalent to $X$ being unicoherent.

LEMma 1. Let $X$ denote the inverse limit of an inverse system $\left\{X_{\lambda}, f_{\lambda_{\mu}}, \Lambda\right\}$ of compact Hausdorff spaces $X_{\lambda}$. If $X_{\lambda}$ has no more than l components (where $l<\infty$ is fixed) for each $\lambda \in \Lambda$, then $X$ has no more than $l$ components.

Presented in part to the Society, January 22, 1970 under the title Inverse limits, multicoherence, and hyperspaces; received by the editors June 17, 1970.

AMS 1969 subject classifications. Primary 5425; Secondary 5455.

Key words and phrases. $A$-triodic, chainable continuum, decomposable, dendrite, dendroid, end point, hereditary unicoherence, monotone map, opposite end points, multicoherence.

(1) This work was partially supported by National Research Council Grant no. A 7346

Copyright (C) 1971, American Mathematical Society 
Proof. Suppose that $X$ has more than $l$ components and let $K_{1}, K_{2}, \ldots, K_{l+1}$ be $l+1$ distinct components of $X$. For each $i=1,2, \ldots, l+1$ and $j=1,2, \ldots, l+1$ with $i \neq j$ let $\Lambda_{i, j}=\left\{\lambda \in \Lambda: \Pi_{\lambda}\left(K_{i}\right)\right.$ and $\Pi_{\lambda}\left(K_{j}\right)$ lie in the same component of $\left.X_{\lambda}\right\}$. Since for each $\lambda \in \Lambda$ there exist two of the components $K_{1}, K_{2}, \ldots, K_{l+1}$ that project down into only one component of $X_{\lambda},\left\{\Lambda_{i, j}: 1 \leqq i \leqq l+1,1 \leqq j \leqq l+1\right.$, and $i \neq j\}=\Lambda$. Since there are only a finite number of the sets $\Lambda_{i, j}$, at least one of them, say $\Lambda_{i_{0}, j_{0}}$, is cofinal in $\Lambda$. It follows from 2.1 of $\left[4\right.$, p. 234] that, for each $\lambda \in \Lambda, \Pi_{\lambda}\left(K_{i_{0}}\right)$ and $\Pi_{\lambda}\left(K_{j_{0}}\right)$ are in the same component $C_{\lambda}$ of $X_{\lambda}$. Since $K_{i_{0}}$ is the inverse limit of the inverse system $\left\{\Pi_{\lambda}\left(K_{i_{0}}\right), f_{\lambda \mu} \mid \Pi_{\lambda}\left(K_{i_{0}}\right), \Lambda\right\}$ and $K_{j_{0}}$ is the inverse limit of the inverse system $\left\{\Pi_{\lambda}\left(K_{j_{0}}\right), f_{\lambda \mu} \mid \Pi_{\lambda}\left(K_{j_{0}}\right), \Lambda\right\}$ (see 2.8 of [4, p. 235]), $K_{i_{0}}$ and $K_{j_{0}}$ are each contained in the inverse limit of the inverse system $\left\{C_{\lambda}, f_{\lambda \mu} \mid C_{\lambda}, \Lambda\right\}$. Since the sets $C_{\lambda}$ are compact and connected, their inverse limit is connected $(2.10$ of $[4$, p. 236]) and this contradicts the assumption that $K_{i_{0}} \neq K_{j_{0}}$.

THEOREM 1. Let $X$ denote the inverse limit of an inverse system $\left\{X_{\lambda}, f_{\lambda \mu}, \Lambda\right\}$ of continua $X_{\lambda}$. If $r\left(X_{\lambda}\right) \leqq k$ (where $k<\infty$ is fixed) and each bonding map $f_{\lambda \mu}$ maps $X_{\lambda}$ onto $X_{\mu}$, then $r(X) \leqq k$.

Proof. Let $A$ and $B$ be subcontinua of $X$ such that $A \cup B=X$ and let $C=A \cap B$. Since $\Pi_{\lambda}$ maps $X$ onto $X_{\lambda}$ (see 2.6 of $\left[4\right.$, p. 235]) and $r\left(X_{i}\right) \leqq k$ for each $i=1,2, \ldots$, $\Pi_{\lambda}(A) \cap \Pi_{\lambda}(B)$ has no more than $k+1$ components. Applying 2.9 of [4, p. 235] and Lemma 1 above, we see that $C$ has no more than $k+1$ components. Hence, $r(X) \leqq k$.

REMARK. The inequalities in Theorem 1 cannot be strengthened to equalities; for the dyadic solenoid, the inverse limit of circles with the onto bonding maps $z \rightarrow z^{2}$, is well-known to be indecomposable and therefore unicoherent. Even a locally connected inverse limit space may be of strictly smaller multicoherence degree than each of the factor spaces. This is easily deduced from example (1) of $[9$, p. 203] and Theorem 1 of [8].

Corollary 1. Let $X$ denote the inverse limit of the inverse system $\left\{X_{\lambda}, f_{\lambda \mu}, \Lambda\right\}$ of continua $X_{\lambda}$. If $X_{\lambda}$ is unicoherent and each bonding map $f_{\lambda \mu}$ maps $X_{\lambda}$ onto $X_{\mu}$, then $X$ is unicoherent.

REMARK. Without ontoness of the bonding maps the inverse limit of unicoherent continua need not be unicoherent (see the Example on p. 412 of [11]). However, if each space is a unicoherent locally connected metric continuum, then the inverse limit is unicoherent (without assuming the bonding maps are onto). To see this, note that under the assumptions above each space has property (b) [14, p. 228] which is equivalent to its first Čech cohomology group (over the integers) being zero [5]. Therefore, by the continuity of Čech theory, the inverse limit has property (b) and, thus, is unicoherent [14, p. 227] (note that the inverse limit space may not be locally connected). I thank Professor John Isbell for simplifying my original proof of this. 
The next theorem gives sufficient conditions in terms of the bonding maps in order that the degree of multicoherence be preserved. Capel's results on arcs and simple closed curves $[4$, p. 244] can be easily obtained from it. Recall that a continuous function $f$ from a continuum $Y$ into a continuum $Z$ is said to be monotone if and only if $f^{-1}(f(y))$ is a continuum for each $y \in Y$.

THEOREM 2. Let $X$ denote the inverse limit of the inverse system $\left\{X_{\lambda}, f_{\lambda \mu}, \Lambda\right\}$ of continua $X_{\lambda}$. If $r\left(X_{\lambda}\right)=k$ (where $k<\infty$ is fixed) for all $\lambda \in \Lambda$ and each bonding map $f_{\lambda \mu}$ is a monotone mapping of $X_{\lambda}$ onto $X_{\mu}$, then $r(X)=k$.

Proof. By Theorem 1 above, $r(X) \leqq k$. Let $\lambda_{0} \in \Lambda$. Use 4.2 of $[4$, p. 241] and 8.62 of $[14$, p. 154$]$ to conclude that $r\left(X_{\lambda_{0}}\right) \leqq r(X)$, i.e., $k \leqq r(X)$.

The proof of Theorem 3 below is immediate from 2.8 of $[4$, p. 235] and Theorem 1 above.

THEOREM 3. Let $X$ denote the inverse limit of an inverse system $\left\{X_{\lambda}, f_{\lambda \mu}, \Lambda\right\}$ of compact Hausdorff spaces $X_{\lambda}$. If each subcontinuum of $X_{\lambda}$ has multicoherence degree $\leqq k$ (where $k<\infty$ is fixed) for all $\lambda \in \Lambda$, then each subcontinuum of $X$ has multicoherence degree $\leqq k$. Thus, if $X_{\lambda}$ is hereditarily unicoherent for all $\lambda \in \Lambda$, then $X$ is hereditarily unicoherent.

Throughout the rest of this paper we consider only countable inverse systems indexed by the natural numbers. For convenience we denote the bonding map from the $(i+1)$ st space $X_{i+1}$ to the $i$ th space $X_{i}$ by $f_{i}$ and we denote the inverse sequence by $\left\{X_{i}, f_{i}\right\}_{i=1}^{\infty}$.

We now apply some of the theorems above to inverse limits of dendroids and dendrites. A dendroid is an arcwise connected metric continuum such that each subcontinuum is unicoherent. A dendrite $[14$, p. 88$]$ is a locally connected metric continuum which contains no simple closed curve.

THEOREM 4. Let $X$ denote the inverse limit of an inverse sequence $\left\{D_{i}, f_{i}\right\}_{i=1}^{\infty}$ where $D_{\imath}$ is a dendroid for each $i=1,2, \ldots$.

1. If $X$ is arcwise connected, then $X$ is a dendroid or a singleton.

2. If $X$ is locally connected, then $X$ is a dendrite or a singleton.

3. If $D_{i}$ is a dendrite and $f_{i}$ is a monotone mapping of $D_{i+1}$ into $D_{i}$ for each $i=1,2, \ldots$, then $X$ is a dendrite or a singleton.

Proof. Without using any of the hypotheses in 1, 2, or 3, we know from Theorem 3 that each subcontinuum of $X$ is unicoherent. This proves 1 ; it also proves 2 since a locally connected dendroid is a dendrite. To prove 3 note that, by 2.8 of [4, p. 235], $X$ is the inverse limit of the inverse sequence $\left\{\Pi_{i}(X), g_{i}\right\}_{i=1}^{\infty}$ where $g_{i}=f_{i} \mid \Pi_{i+1}(X)$ for each $i=1,2, \ldots$ It is not difficult to verify that, since every subcontinuum of $D_{i}$ is unicoherent for each $i=1,2, \ldots, g_{i}$ is monotone for each $i=1,2, \ldots$ Also $g_{i}$ maps $\Pi_{i+1}(X)$ onto $\Pi_{i}(X)$ (see 2.8 of $[4$, p. 235]). Therefore, by 4.3 of $[4$, p. 241], $X$ is locally connected. Part 3 now follows from part 2 . 
In [4, p. 244] Capel proved that the inverse limit space of an inverse sequence of arcs with monotone onto bonding maps is an arc. Fort and Segal [7, p. 256] generalized Capel's result by requiring not that the bonding maps be monotone but only that the inverse limit space be locally connected. We now (Theorem 5) extend the result of Fort and Segal concerning arcs by only requiring that the inverse limit space be arcwise connected. Most of the proof of this result appears in [11]. However, we include here the following lemma which verifies the second sentence of the proof in [11].

LEMma 2. If a dendroid is not an arc, then it contains a triod.

Proof. Let $X$ be a dendroid. Note that if two arcs in $X$ intersect, then their union is either an arc or contains a triod. Suppose $X$ does not contain a triod. Then a countable dense subset of $X$ can be used to construct a monotone increasing sequence of arcs whose union is a dense subset of $X$. By Lemma 3 of [3] such a union must be contained in an arc, and it now follows that $X$ is an arc. This establishes a contradiction and completes the proof of the lemma.

TheOREM 5. Let $X$ denote the inverse limit of an inverse sequence $\left\{A_{i}, f_{i}\right\}_{i=1}^{\infty}$ where $A_{i}$ is an arc for each $i=1,2, \ldots$ If $X$ is arcwise connected, then $X$ is an arc or $a$ singleton.

Proof. By Theorem 4, $X$ is a dendroid or a singleton. If $X$ is neither an arc nor a singleton then, by Lemma $2, X$ contains a triod $T$. Using 2.8 and 2.11 of [4] we see that $T$ is an inverse limit of arcs, a contradiction (see the remark below).

REMARK. Using Lemma 1 above it is easy to give a direct proof that a triod is not an inverse limit of arcs (we could also use Theorem 4 of [7]). There is a wellknown theorem (which could also be used to justify the contradiction at the end of the proof of Theorem 5) that a metric continuum is chainable if and only if it is the inverse limit of an inverse sequence of arcs. Hence, Theorem 5 proves that the only arcwise connected chainable metric continuum is an arc. See "Added in proof".

3. Arcwise connected circle-like continua. In this section we use material from $\$ 2$ of this paper and from [12] to obtain information about the structure of arcwise connected inverse limits of circles (with onto bonding maps). We point out that a very simple proof of the second part of Theorem 4 of [7] (which says that a locally connected inverse limit of circles with onto bonding maps is a circle) can be obtained using some of our results in $\$ 2$.

We will use the terms simple closed curve and circle interchangeably to mean a space homeomorphic to $\{z$ in the plane: $|z|=1\}$.

THEOREM 6. Let $X$ denote the inverse limit of an inverse sequence $\left\{S_{i}, f_{i}\right\}_{i=1}^{\infty}$ where $S_{i}$ is a simple closed curve and $f_{i}$ maps $S_{i+1}$ onto $S_{i}$ for all $i=1,2, \ldots$ If $X$ is arcwise connected, then either 
1. $X$ is a simple closed curve, or

2. $X$ can be written in the form $A \cup C$ where $A$ is an arc, $C$ is a chainable metric continuum with exactly two arc components, and $A \cap C$ is exactly the two noncut points of $A$. Furthermore, the two points of $A \cap C$ are opposite end points of $C$.

Conversely, a metric continuum satisfying 1 or 2 is an arcwise connected inverse limit of circles with onto bonding maps.

Proof. First note that by 2.8 and 2.11 of [4]

(a) $X$ is $a$-triodic, and

(b) each proper subcontinuum of $X$ is chainable.

By Theorem $1, r(X) \leqq 1$. Suppose $r(X)=0$. Then it follows from (b) that $X$ is a dendroid. Thus, by Lemma 2 and (a), $X$ must be an arc. However, a simple application of Lemma 1 (or direct use of Theorem 4 of [7]) shows that $X$ cannot be an arc. Thus, $r(X) \neq 0$ which implies that $r(X)=1$. Let $E$ and $F$ be subcontinua of $X$ such that $X=E \cup F$ and $E \cap F$ is not connected. Assume first that $E$ and $F$ are each arcwise connected. Then, from (b) and Theorem 5 above, $E$ and $F$ are each arcs. Thus, since $E \cap F$ is not connected, $E \cup F$ contains a simple closed curve $S$. But, by (b), $S$ must equal $X$ which proves that $X$ is a simple closed curve. From now on we assume that at least one of $E$ and $F$ is not arcwise connected, say $F$. Suppose $F$ had three (or possibly more) distinct arc components $F_{1}, F_{2}$ and $F_{3}$. Let $\beta$ be an arc in $X$ with one noncut point in $F_{1}$ and the other in $F_{2}$ and let $\gamma$ be an arc in $X$ with the same noncut point in $F_{2}$ as $\beta$ and with the other noncut point in $F_{3}$. If $\beta \cup \gamma=X$, then the non-unicoherence of $X$ would imply $X$ contained, and therefore was, a simple closed curve; this would contradict the assumption about $F$. Hence, $\beta \cup \gamma$ is a proper subcontinuum of $X$. From (b) above and the local connectivity of $\beta \cup \gamma$, it now follows that $\beta \cup \gamma$ is an arc. Now since each component of $F \cap(\beta \cup \gamma)$ is a subcontinuum of $\beta \cup \gamma$, each such component is an arc. Hence, there are at least three such components, so $r(F \cup(\beta \cup \gamma)) \geqq 2$ which contradicts the proven fact that $r(X)=1$ (clearly $F \cup(\beta \cup \gamma$ ), not being unicoherent, cannot be chainable; so by (b), $F \cup(\beta \cup \gamma)=X)$. Since $F$ is not arcwise connected, we have now proved that $F$ has exactly two arc components. Also, $F$ being a proper subcontinuum of $X, F$ is a chainable metric continuum. Let $C=F$. To show how to pick $A$ and verify the properties of $A \cap C$ we use the following lemma which is the main result of [12].

LEMMA 3. If a chainable metric continuum has exactly two arc components, then one of them is an arc and the other is a half-ray (i.e., homeomorphic to $[0, \infty)$ ).

So, by Lemma 3, one of the arc components of $C$ is an arc and the other a halfray. Denote the one which is an arc by $I$ and the one which is a half-ray by $H$. Let $h$ denote the noncut point of $H$. Let $\alpha$ be an arc in $X$ with one noncut point being $h$ and the other a point of $I$. Using (a) and the fact that $H$ is not contained in an arc, it is easy to see that $\alpha \cap H=\{h\}$. It is also easy to see that $\alpha \cap I$ is an arc or a point which includes at least one noncut point of $I$. If $\alpha \cap I$ includes only one 
noncut point of $I$, then let $e$ denote that noncut point. If $\alpha \cap I$ includes both noncut points of $I$, then let $e$ denote the noncut point of $I$ which is a cut point of $\alpha$. Let $A$ be the subarc of $\alpha$ with $e$ and $h$ as its two noncut points. Clearly, $A \cap C$ is exactly the two noncut points of $A$. We now show that $h$ and $e$ are opposite end points of $C$ (in the sense of [1, p. 661]). Using that $C$ is $a$-triodic [1, p. 653] (in particular, we use that $\bar{H}$ contains at least one noncut point of $I$ ) and Lemma 3 above it is easy to verify (A) of $[1$, p. 660] for the point $h$ and therefore, by Theorem 13 of [1, p. 661], $h$ is an end point of $C$. Furthermore, if $\bar{H} \supset I$, then Lemma 3 above and (A) and Theorem 13 of [1] can be used again to show that each of the noncut points of $I$ (thus, in particular, $e$ ) are end points of $C$; it then follows, using Theorem 14 of [1, p. 661], that $h$ and $e$ are opposite end points of $C$. Now we consider the case when $\bar{H} \neq I$. If $e \in \bar{H}$, then $A \cup \bar{H}$ would not be unicoherent (because $A \cap \bar{H}$ would equal $\{h, e\}$ ) but would be a proper (because $\bar{H} \nsupseteq I$ ) subcontinuum of $X$, contradicting (b) above. Thus, $e \notin \bar{H}$. It is now easy to see that

$\left(^{*}\right)$ any subcontinuum of $C$ containing $e$ and some point of $H$, must contain $I$.

Using (*) it is easy to verify (A) of [1, p. 660] for the point $e$ and thus, by Theorem 13 of [1, p. 661], $e$ is an end point of $C$. It is also easy to see, using $(*)$, that $C$ is irreducible between $h$ and $e$ and therefore, by Theorem 14 of [1, p. 661], $h$ and $e$ are opposite end points of $C$. This completes the proof of the necessity of conditions 1 and 2 of the theorem.

Conversely, let $X$ be a metric continuum satisfying 2 (the case when $X$ satisfies 1 is trivial) of Theorem 6 . By Lemma 3 one of the arc components of $C$, denoted as before by $H$, is a half-ray and the other, denoted by $I$, is an arc. Since the two points of $A \cap C$ are opposite end points of $C$, one of the points in $A \cap C$ is the noncut point $h$ of $H$ and the other is a noncut point $e$ of $I$. From the fact that $A$ intersects both $H$ and $I$ it follows easily that $X$ is arcwise connected. We now show that $X$ is an inverse limit of circles with onto bonding maps. Let $\varepsilon>0$. Let $U=\left\{U_{1}, \ldots, U_{n}\right\}$ be a finite number of open subsets of $C$ such that $U$ is an $\varepsilon$-chain from $h$ to $e$ covering $C[1$, p. 661]. Let $|N(U)|$ denote the (geometric) realization of the nerve of $U$; note that $|N(U)|$ is topologically an arc. Let $\Psi: C \rightarrow|N(U)|$ be a canonical mapping relative to $U\left[6, \mathrm{p}\right.$. 286]. Since $h \in\left(U_{1}-U_{2}\right)$ and $e \in\left(U_{n}-U_{n-1}\right)$, $\Psi(h)$ is one of the noncut points of $|N(U)|$ and $\Psi(e)$ is the other noncut point of $|N(U)|$ (this follows directly the definition of canonical [6, p. 286]). It is also easy to see that, since diam $\left(U_{i}\right)<\varepsilon$ for all $i=1, \ldots, n, \Psi$ is an $\varepsilon$-mapping. Let $S^{1}$ denote the unit circle in the plane, let $S_{+}^{1}=\left\{(x, y) \in S^{1}: y \geqq 0\right\}$, and let $S_{-}^{1}=\left\{(x, y) \in S^{1}: y \leqq 0\right\}$. By following $\Psi$ with a homeomorphism of $|N(U)|$ onto $S_{+}^{1}$, we obtain an $\varepsilon$-mapping $f$ of $C$ onto $S_{+}^{1}$; without loss of generality we may assume $f(h)=(-1,0)$ and $f(e)=(1,0)$. Since $A$ is an arc with noncut points $h$ and $e$, there is a homeomorphism $g$ of $A$ onto $S_{-}^{1}$ such that $g(h)=(-1,0)$ and $g(e)$ $=(1,0)$. The function which is $f$ on $C$ and $g$ on $A$ is an $\varepsilon$-mapping of $X$ onto $S^{1}$. Since $\varepsilon$ was arbitrary, the fact that $X$ is an inverse limit of circles with onto bonding maps now follows from Theorem 1 of [8]: This completes the proof of Theorem 6. 
REMARK. Just as there are uncountably many topological types of chainable metric continua with exactly two arc components [12], so there are uncountably many topological types of arcwise connected inverse limits of circles. However, Theorem 6 above and Theorem 1 of [12] (stated as Lemma 3 above) determine to a large extent the structure of such continua.

COROLlaRY. If $X$ is an arcwise connected inverse limit (with onto bonding maps) of circles, then $X$ is embeddable in the plane.

Proof. By Theorem 6 (see the first remark below) such an $X$ is decomposable and therefore, by Theorem 2 and Theorem 3 of [13], $X$ is embeddable in the plane.

REMARK. Theorem 6 states a specific type of decomposition for such an $X$. However, any arcwise connected metric continuum is decomposable (this is easily seen by using an argument involving composants). This general fact could have been used here.

REMARK. Two other proofs (these depend on the geometric character of $X$ as given in Theorem 6) of the Corollary above can be given. Theorem 6 could be used to verify the hypotheses of Theorem 4 of [2] and thus prove that $X$ is embeddable in the plane. Yet another proof could be based on Proposition 2 of [10] by using Theorem 6 to show directly that $H^{1}(X, Z)$ is infinite cyclic.

Added in proof. It was pointed out to me recently by W. S. Mahavier that Theorem 5 in the form of the Remark following it was noted in a paper by him and M. Friedberg entitled Semigroups on chainable and circle-like continua, Math. Z. 106 (1968), 159-161.

\section{REFERENCES}

1. R. H. Bing, Snake-like continua, Duke Math. J. 18 (1951), 653-663. MR 13, 265.

2. - Embedding circle-like continua in the plane, Canad. J. Math. 14 (1962), 113-128. MR 24 \#A1712.

3. K. Borsuk, A theorem on fixed points, Bull. Polon. Acad. Sci. Cl. III 2 (1954), 17-20. MR 16, 275.

4. C. E. Capel, Inverse limit spaces, Duke Math. J. 21 (1954), 233-245. MR 15, 976.

5. C. H. Dowker, Mapping theorems for non-compact spaces, Amer. J. Math. 69 (1947), 200-242. MR 8, 594.

6. Samuel Eilenberg and Norman Steenrod, Foundations of algebraic topology, Princeton Univ. Press, Princeton, N. J., 1952. MR 14, 398.

7. M. K. Fort, Jr. and Jack Segal, Local connectedness of inverse limit spaces, Duke Math. J. 28 (1961), 253-259. MR 23 \#A3549.

8. Sibe Mardešić and Jack Segal, e-mappings onto polyhedra, Trans. Amer. Math. Soc. 109 (1963), 146-164. MR 28 \#1592.

9. C. N. Maxwell, An order relation among topological spaces, Trans. Amer. Math. Soc. 99 (1961), 201-204. MR 22 \#11374.

10. M. C. McCord, Embedding P-like compacta in manifolds, Canad. J. Math. 19 (1967), 321-332. MR 35 \#3669.

11. S. B. Nadler, Jr., Inverse limits and multicoherence, Bull. Amer. Math. Soc. 76 (1970), 411-414. 
12. S. B. Nadler, Jr. Arc components of certain chainable continua, Canad. Math. Bull. (to appear).

13. J. T. Rogers, Jr., Mapping the pseudo-arc onto circle-like, self-entwined continua, Michigan Math. J. 17 (1970), 91-96.

14. G. T. Whyburn, Analytic topology, Amer. Math. Soc. Colloq. Publ., vol. 28, Amer. Math. Soc., Providence, R. I., 1942. MR 4, 86.

DALHOUSIE UNIVERSITY,

Halifax, Nova Scotia, Canada

LOYOLA UNIVERSITY,

New OrLeans, Louisiana 70118 